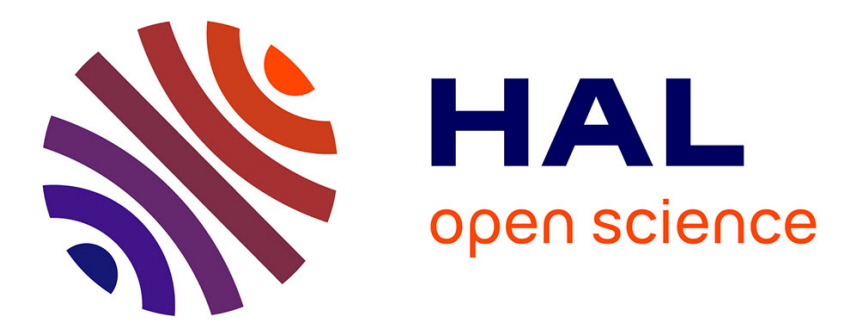

\title{
Unstructured finite element-based digital image correlation with enhanced management of quadrature and lens distortions
}

Jean-Emmanuel Pierré, Jean-Charles Passieux, Jean-Noël Périé, Florian Bugarin, Laurent Robert

\section{To cite this version:}

Jean-Emmanuel Pierré, Jean-Charles Passieux, Jean-Noël Périé, Florian Bugarin, Laurent Robert. Unstructured finite element-based digital image correlation with enhanced management of quadrature and lens distortions. Optics and Lasers in Engineering, 2016, 77, pp.44-53. 10.1016/j.optlaseng.2015.07.008 . hal-01217885

\section{HAL Id: hal-01217885 \\ https://hal.science/hal-01217885}

Submitted on 20 Oct 2015

HAL is a multi-disciplinary open access archive for the deposit and dissemination of scientific research documents, whether they are published or not. The documents may come from teaching and research institutions in France or abroad, or from public or private research centers.
L'archive ouverte pluridisciplinaire HAL, est destinée au dépôt et à la diffusion de documents scientifiques de niveau recherche, publiés ou non, émanant des établissements d'enseignement et de recherche français ou étrangers, des laboratoires publics ou privés. 


\title{
Unstructured Finite Element-based Digital Image Correlation with enhanced management of quadrature and lens distortions
}

\author{
J.-E. Pierré, J.-C. Passieux, J.-N. Périé, F. Bugarin, L. Robert ${ }^{\dagger}$ \\ Université de Toulouse - Institut Clément Ader - INSA/UPS/Mines Albi/ISAE, 3, rue Caroline Aigle \\ F-31400 Toulouse, France \\ $\dagger$ This paper is dedicated to the memory of Dr. Laurent Robert, a friend and colleague, whom we miss.
}

\begin{abstract}
Like subset-based methods, the very first finite element versions of digital image correlation were closely related to the regular structure of images, as they were based on regular quadrilateral elements corresponding to an integer number of pixels. The use of unstructured meshes, to exploit the full potential of FE-DIC in structural mechanics, is now widespread. Most of the time, the formulation, the quadrature and the definition of the region of interest still rely on the pixels grid. In this paper, a formulation in the physical coordinate system and not in the image frame is proposed for $2 \mathrm{D}$ digital image correlation. In addition to a more precise definition of the region of interest, it allows the use of a more accurate quadrature rule. It is also shown that lens distortions can be successfully taken into account directly with such a formalism.
\end{abstract}

Keywords: Digital Image Correlation, Finite Element, Distortion, Pin-hole

\section{Introduction}

Digital Image Correlation (DIC) is now extensively used in the field of experimental mechanics for identification and validation purposes. The basic principle of DIC methods is to look for a displacement field that ensures at best the grey level conservation between two images. Most industrial codes are based on subset based approaches [1, 2. Since Digital Image Correlation was originally developed by the community of image analysis [1, the derived methods were (and are still) closely related to the regular structure of the image. Indeed, original methods rely on a piecewise description of the displacement in several square (or at least rectangular) subsets corresponding to an integer number of pixels, called Zones Of Interest $(Z O I)$ [2].

Conversely, many recent developments rather rely on global descriptions of the unknown displacement. The latter is thus defined in the whole Region Of Interest (ROI). A certain regularity of the displacement field can then be enforced a priori. Several types of decomposition of the displacement field have been proposed. For instance, the reader may refer to [3, 4, 5, 6, 7, 8, 9, 10, to name a few.

In this paper, we focus on a Finite Element approach to DIC (FE-DIC [5, 6]). It was shown that this kind of approach has many advantages in the context of continuum solid mechanics and especially for the experiment/simulation dialog [11]. This kind of tool is particularly appealing in the context of a Finite Element Model Updating approach based on displacement fields (FEMU-U [12, 13, 14]). It is then tempting to use the (optimized) simulation mesh to perform the FE-DIC measurements [15]. 
However, the very first developments [5, 16, 17] were still based on regular quadrilateral elements (denoted Q4) corresponding to an integer number of pixels. The use of unstructured meshes is now widespread [13, 18, 19, 15, 20, 21] to take advantage of the full potential of FE-DIC. Most of the previous references still rely on the pixels, since their integration scheme is based on pixels and since the definition of the $R O I$ is also defined by a set of pixels.

Recently, a new formulation of the DIC problem in the physical space has been proposed in the context of stereo-DIC [22, 18]. It is based on a kinematic model (FE mesh, NURBS patch...) in the world coordinate system along with a projective model. This paper investigate the effect of such a formulation in the context of 2D FE-DIC. In practice, the 3D mesh of the specimen skin is used. Its position in space is defined so that its projection(s) on the reference image(s) matches at best different amers. The idea is then to search for the displacement, directly expressed in the physical space, minimising a continuous problem associated to the grey level conservation. Images are only considered as simple data. The algorithm compares, for any point in any element, the grey levels corresponding to its projection in different images. This paper shows that such a formulation is of interest for $2 \mathrm{D}$ FE-DIC for the two following reasons:

1. the assembly of the numerical operators requires an appropriate quadrature rule. In opposition with standard approaches, for which the reference image is used to define both the integration technique and the $R O I$, here, a dedicated surface quadrature technique in the world reference system is defined. The efficiency of those two integration methods will be compared;

2. as for stereo DIC algorithms, distortions can be taken into account during the measurement. Even if it seems less natural to resort to calibration in this context, [23] recently shown that it is worth taking distortions into account even for $2 \mathrm{D}$ analysis.

The effect of lens distortions will be considered here but not the one due to out-of-plane motions [24, 25, 26, 27]. In general, the latter cannot be taken into account in 2D-DIC without strong kinematic assumptions. For instance, it is possible when considering a rigid-body motion of the observed object [10, 28]. With a single camera, it is difficult to distinguish the effects of out-of-plane motions and strains as it is explained in [25].

In this article, a synthetic image, subjected to an in plane (large) translation, is considered. The 2D displacement field is measured with a given mesh and a calibration model.

Section 2 presents the usual quadrature method. This method considers a Region of Interest $(R O I)$ which is simply a part of the reference image. Section 3 introduces a mesh based method quadrature, better suited for a Finite Element DIC Method. The two previous approaches are compared in Sect. 4 in terms of measurement uncertainties. At this stage, a simple projector is used. Finally, in Sect. 5, the way distortions can be taken into account into the mesh based integration is presented. A pin-hole model of camera is considered in order to evaluate the gain with respect to measurement uncertainties.

\section{Quadrature at the pixels}

\subsection{Formulation}

The optical flow problem between the reference state image $f$ and the deformed state image $g$ reads:

$$
f(\mathbf{x})=g(\mathbf{y})
$$


where $\mathbf{x}$ and $\mathbf{y}$ are the pixels positions. The latter problem is usually written in a weak form over a region called Region of Interest $(R O I)$. The $R O I$ is often defined as part of an image, and more precisely, a part of the reference state image $f$. In this equation, $\mathbf{y}=\mathbf{x}+\mathbf{u}(\mathbf{x})$ and the problem reads:

$$
\mathbf{u}^{\star}=\underset{\mathbf{u} \in \mathcal{L}^{2}(R O I)}{\operatorname{argmin}} \int_{R O I}[f(\mathbf{x})-\bar{g}(\mathbf{x}+\mathbf{u}(\mathbf{x}))]^{2} d \mathbf{x}
$$

with $\bar{g}$ the deformed state image with corrected mean value and standard deviation, in order to take into account offset and scale changes in illumination [29, 30, 31, 10].

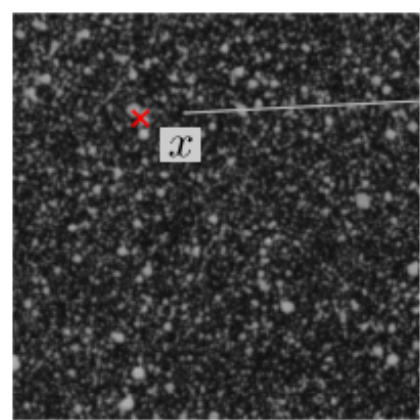

reference image $f$

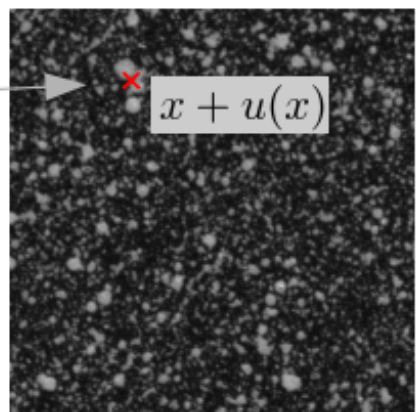

deformed image $g$

Figure 1: Reference image with a point $\mathbf{x}$ (left) and deformed image with the corresponding point $\mathbf{x}+\mathbf{u}(\mathbf{x})$ (right)

This problem being non-linear, a first order Taylor expansion of $\bar{g}$ is performed with respect to the displacement correction $\delta \mathbf{u}=\mathbf{u}-\mathbf{u}_{0}$ given an initial estimate $\mathbf{u}_{0}$. Therefore, the linearised problem reads [6, 15]

$$
\mathbf{u}^{\star}=\underset{\mathbf{u} \in \mathcal{L}^{2}(R O I)}{\operatorname{argmin}} \int_{R O I}\left[\nabla f^{T} \delta \mathbf{u}(\mathbf{x})-r(\mathbf{x})\right]^{2} d \mathbf{x}
$$

where $r(\mathbf{x})=f(\mathbf{x})-\bar{g}\left(\mathbf{x}+\mathbf{u}_{0}(\mathbf{x})\right)$ is the gray level residual field and $\nabla f$ the gradient of the reference image $f$ estimated by finite differences. This problem has got a variational form:

Find $\delta \mathbf{u} \in \mathcal{L}^{2}(R O I)$, such that:

$$
\begin{gathered}
a(\delta \mathbf{u}, \mathbf{v})=l(\mathbf{v}) \quad \forall \mathbf{v} \in \mathcal{L}^{2}(R O I) \\
\text { with }\left\{\begin{array}{c}
a(\mathbf{u}, \mathbf{v})=\int_{R O I} \mathbf{u}^{\top}(\mathbf{x}) \nabla f(\mathbf{x}) \nabla f^{\top}(\mathbf{x}) \mathbf{v}(\mathbf{x}) d \mathbf{x} \\
l(\mathbf{v})=\int_{R O I} \mathbf{v}^{\top}(\mathbf{x}) \nabla f(\mathbf{x}) r(\mathbf{x}) d \mathbf{x}
\end{array}\right.
\end{gathered}
$$

In this work, the interpolation of the unknown displacement $\delta \mathbf{u}$ is based on finite elements [5, 6] such that $\delta \mathbf{u}(\mathbf{x})=\sum_{i} \mathbf{N}_{i}(\mathbf{x}) q_{i}$ with $q_{i}$ the degrees of freedom and $\mathbf{N}_{i}$ the shape functions. Then, the linearised DIC problem reads:

$$
\mathbf{M}^{D I C} \mathbf{q}=\mathbf{b}^{D I C} \text { with: }\left\{\begin{aligned}
\mathbf{M}_{i j}^{D I C} & =a\left(\mathbf{N}_{i}, \mathbf{N}_{j}\right) \\
\mathbf{b}_{i}^{D I C} & =l\left(\mathbf{N}_{i}\right)
\end{aligned}\right.
$$


For a sufficiently regular image gradient, it can be shown that the correlation matrix $\mathbf{M}^{D I C}$ is symmetric definite positive [32]. Another interesting result is that $\mathbf{M}^{D I C}$ only depends on the initial data, so its computation is done only once for each time step.

\subsection{Quadrature}

In this section, a classical quadrature rule based on the image is explained to compute $\mathbf{M}^{D I C}$ and $\mathbf{b}^{D I C}$. Indeed, the integrals of equations (6) are approximated by:

$$
\int_{R O I} \psi(\mathbf{x}) d \mathbf{x} \approx \sum_{e \in N_{e}} \sum_{k \in I_{e}} \omega_{k}^{e} \psi\left(\mathbf{x}_{k}^{e}\right)
$$

where $\mathbf{x}_{k}^{e}$ and $\omega_{k}^{e}$ are the quadrature points and weight coefficients of element $e$ respectively; $N_{e}$ being the number of elements. $I_{e}$ the set of quadrature points $\mathbf{x}_{k}^{e}$ is detailed here.

As stated previously, the Region of Interest $(R O I)$ is given here by a set of pixels. The nodes of element $e$ are first projected in the reference image and the element is reconstructed in the image plane. For instance, with linear elements, it means that the edges are assumed to remain straight in the image plane (this assumption is actually not true when considering lens distortions, which is the object of Sect. 50. In this case, $I_{e}$ is the set of pixels whose center belongs to element $e$ and $\mathbf{x}_{k}^{e}$ is the center of the pixel. The associated weight coefficients are the area of one pixel, namely $\omega_{k}^{e}=1$.

Since $\mathbf{x}_{k}^{e}$ is a pixel of the reference image $f$, this means that $f$ is also the reference space for the integration. Finally, the computation of the left hand side of equation (6), for instance, reads:

$$
a\left(\mathbf{N}_{i}, \mathbf{N}_{j}\right) \approx \sum_{e \in N_{e}} \sum_{k \in I_{e}} \mathbf{N}_{i}^{\top}\left(\mathbf{x}_{k}^{e}\right) \nabla f\left(\mathbf{x}_{k}^{e}\right) \nabla f^{\top}\left(\mathbf{x}_{k}^{e}\right) \mathbf{N}_{j}\left(\mathbf{x}_{k}^{e}\right)
$$

The position of the quadrature points being known in the real coordinate system, another issue concerns the evaluation of the shape functions $\mathbf{N}\left(\mathbf{x}_{k}^{e}\right)$. Indeed, in expression (8), the shape functions $\mathbf{N}_{i}$ has to be evaluated at pixel $\mathbf{x}_{k}^{e}$, knowing its coordinates in the global system $(x, y)$ (cf. Fig. 2). But with the Finite Element Method, an iso-parametric representation of the element is used, and the shape functions $\mathbf{N}$ are known in the reference coordinate system $(\xi, \eta)[21]$.

These basis functions are used to describe the unknown displacement field but also the geometry of the element. Indeed, knowing the real positions $\mathbf{s}_{i}=\left(x_{i}, y_{i}\right)$ of the vertices of element $e$, the mapping between the coordinates $(\xi, \eta)$ of a point $\mathbf{x}^{0}$ in the reference configuration and the coordinates $(x, y)$ of the corresponding point $\mathbf{x}=\phi\left(\mathbf{x}^{0}\right)$ in the real configuration is given by [33] (cf. Fig. 2).

$$
\mathbf{x}=\sum_{i} N_{i}(\xi, \eta) \mathbf{s}_{i}
$$

To compute the elementary DIC operators, the inverse of this mapping has to be determined. Indeed, the coordinates $\left(x_{p}, y_{p}\right)$ of a pixel $\mathbf{x}$ are known in the real configuration, and the coordinates $\left(\xi_{p}, \eta_{p}\right)$ of its corresponding point $\mathbf{x}^{0}$ are searched for. This is performed, in practice, thanks to few iterations of a Newton algorithm on $\phi\left(\xi_{p}, \eta_{p}\right)=\mathbf{x}-\sum N_{i}\left(\xi_{p}, \eta_{p}\right) \mathbf{s}_{i}$

Remark. For the calculation of $r\left(\mathbf{x}_{k}\right), f\left(\mathbf{x}_{k}\right)$ defines the gray level of pixel $\mathbf{x}_{k}$ in the reference state image. In opposition, $\mathbf{x}_{k}+\mathbf{u}\left(\mathbf{x}_{k}\right)$ may not be an integer number of pixels and thus requires to use a gray level interpolation (usually bilinear, bi-cubic or spline) for computing $\bar{g}\left(\mathbf{x}_{k}+\mathbf{u}\left(\mathbf{x}_{k}\right)\right.$ ).

The limitations of such an approach are as follows: 


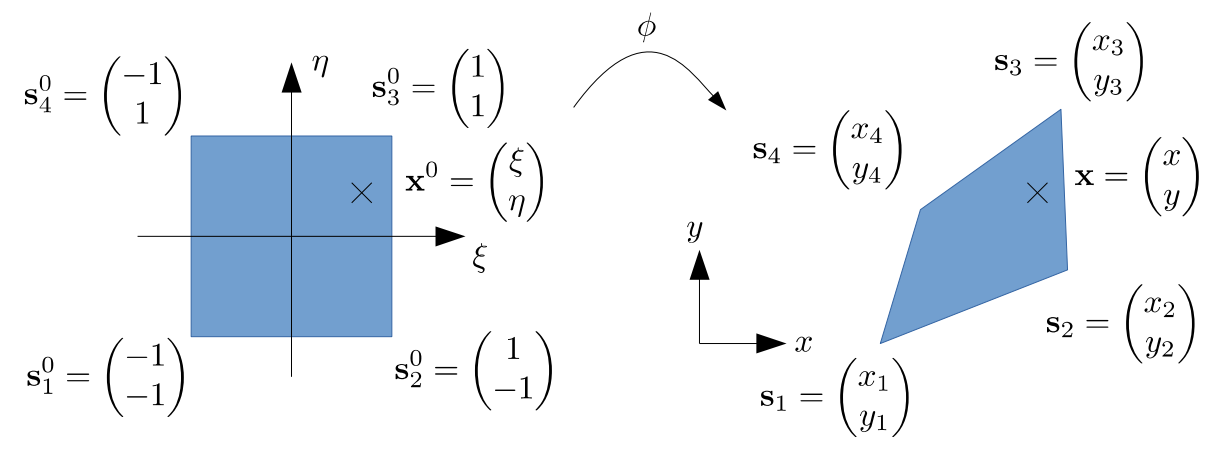

Figure 2: Reference element (left) and real element on a mesh (right) for a quadrangle Q4

- the quadrature rule is not exact even for computing the quadrature of a constant function. This means that the area of an element is not exact ( $c f$. Fig. 3). This yields measurement uncertainties;

- the geometric assumptions are required which may be relevant enough with telecentric lenses, but not in the general case. This is particularly important when quantitative comparison with numerical simulation are searched for.

In the following section, an alternative is presented which does not have these limitations.

\section{"Classical" Pixel Integration}

Mesh: $\Omega$

Projection of the nodes of the mesh:

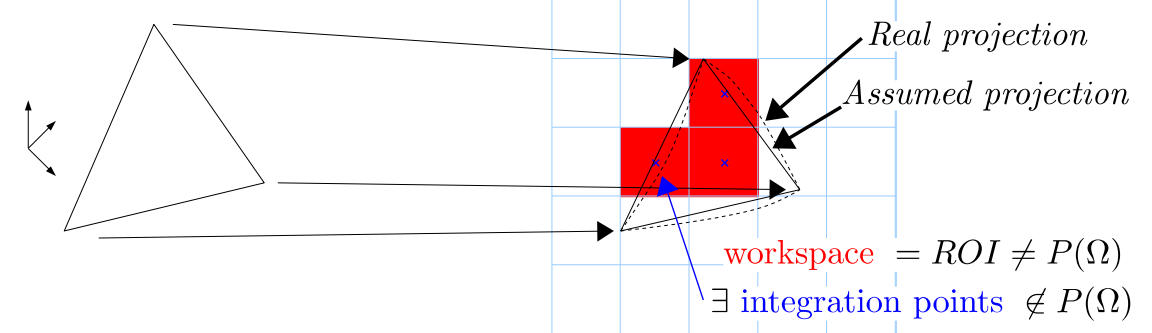

Figure 3: With the classical method the workspace is part of an image and there are some approximations (the computed area, in red, is not exact).

\section{Mesh Based Quadrature}

\subsection{Formulation}

The idea is to compute the integration in the physical space defined by the mesh and not in the $R O I$ positioned in the image. This method considers a projection model, denoted $P$, which maps the coordinates $\mathbf{X}$ (in meter) of a point in the physical space to the coordinates $\mathbf{x}$ (in pixel) of its projection onto the image plane. Different examples of projector will be detailed in Sections 4 and 5.

Assuming that $P$ is known, it is possible to write the gray level conservation equation (1) with:

$$
\left\{\begin{array}{l}
\mathbf{x}=P(\mathbf{X}) \\
\mathbf{y}=P(\mathbf{X}+\mathbf{U}(\mathbf{X}))
\end{array}\right.
$$


where the unknown is the displacement defined in the physical space $\mathbf{U}$. Then, the weak form of the optical flow problem (1) is written as an integral over $\Omega$ which is the domain defined by the model in the physical space and not in the image:

$$
\mathbf{U}^{\star}=\underset{\mathbf{U} \in \mathcal{L}^{2}(\Omega)}{\operatorname{argmin}} \int_{\Omega}[f(P(\mathbf{X}))-\bar{g}(P(\mathbf{X}+\mathbf{U}(\mathbf{X})))]^{2} d \mathbf{X}
$$

Remark. A link between the ROI in the standard FE technique and the 3D domain $\Omega$ could be $R O I=P(\Omega)$. But, as it will be shown in Sections 4 and 5 , in practice, with classical FE-DIC techniques, this equality may not be perfectly verified.

The problem is again linearised by considering a displacement increment $\delta \mathbf{U}=\mathbf{U}-\mathbf{U}_{0}$. This unknown is searched for in an approximation subspace defined in the physical space by the FE interpolation:

$$
\delta \mathbf{U}(\mathbf{X})=\sum_{i} \mathbf{N}_{i}(\mathbf{X}) q_{i}
$$

The variational form thus becomes:

$$
\begin{aligned}
& \text { Find } \mathbf{u} \in \mathcal{L}^{2}(\Omega): a(\mathbf{u}, \mathbf{v})=l(\mathbf{v}) \forall \mathbf{v} \in \mathcal{L}^{2}(\Omega) \text { with: } \\
& \qquad\left\{\begin{array}{c}
a(\mathbf{u}, \mathbf{v})=\int_{\Omega} \mathbf{u}^{\top} \nabla P \nabla f \nabla f^{\top} \nabla P^{\top} \mathbf{v} d \mathbf{X} \\
l(\mathbf{v})=\int_{\Omega} \mathbf{v}^{\top} \nabla P \nabla f r(\mathbf{X}) d \mathbf{X}
\end{array}\right.
\end{aligned}
$$

where the residual map $r(\mathbf{X})$ and the gradients of the projector $\nabla P$ and the reference image $\nabla f$ read:

$$
\left\{\begin{aligned}
r(\mathbf{X}) & =f(P(\mathbf{X}))-\bar{g}\left(P\left(\mathbf{X}+\mathbf{U}_{0}(\mathbf{X})\right)\right) \\
\nabla P & =\nabla P\left(\mathbf{X}+\mathbf{U}_{0}(\mathbf{X})\right) \\
\nabla f & =\nabla f(P(\mathbf{X}))
\end{aligned}\right.
$$

And the correlation system to be solved iteratively is in the same form as before:

$$
\mathbf{M q}=\mathbf{b} \text { with: }\left\{\begin{aligned}
\mathbf{M}_{i j} & =a\left(\mathbf{N}_{i}, \mathbf{N}_{j}\right) \\
\mathbf{b}_{i} & =l\left(\mathbf{N}_{i}\right)
\end{aligned}\right.
$$

It can be seen that the correlation matrix depends on the gradient of the projector ( $c f$. eq. 14). A priori, this operator may depend on the approximation of the unknown $\mathbf{U}_{0}(\mathbf{X})$. As a consequence, correlation matrix $\mathbf{M}$ may also depend on it, which implies that it may be rebuild at each iteration. A discussion on this point will be given in Sections 4 and 5 depending on the type of projector.

Furthermore, the computation of each term of equation (14) requires a gray level interpolation of both reference and deformed images because of the projector: both $P(\mathbf{X})$ and $P\left(\mathbf{X}+\mathbf{U}_{0}(\mathbf{X})\right)$ may not be integer numbers of pixels.

\subsection{Quadrature}

To compute the integrals of $\mathbf{M}$ and $\mathbf{b}$ in the physical space, a quadrature rule based on the mesh is required. As explained previously, the integral of, for instance, the left hand side of 
equation (15), is approximated by:

$$
\begin{array}{r}
a\left(\mathbf{N}_{i}, \mathbf{N}_{j}\right) \approx \sum_{e \in N_{e}} \sum_{k \in I_{e}} \mathbf{N}_{i}^{\top}\left(\mathbf{X}_{k}^{e}\right) \nabla P\left(\mathbf{X}_{k}^{e}\right) \nabla f\left(P\left(\mathbf{X}_{k}^{e}\right)\right) \\
\nabla f^{\top}\left(P\left(\mathbf{X}_{k}^{e}\right)\right) \nabla P^{\top}\left(\mathbf{X}_{k}^{e}\right) \mathbf{N}_{j}\left(\mathbf{X}_{k}^{e}\right)
\end{array}
$$

where $I_{e}$ remains a set of quadrature points $\mathbf{X}_{k}$ associated to weight coefficients $\omega_{k}^{e}$. The difference with Sect. 2, is that the quadrature points are defined in the physical space. Following what is done in FE simulation, the integrals are estimated by:

$$
\int_{\Omega} f(P(\mathbf{X})) d \mathbf{X}=\int_{\phi^{-1}(\Omega)} f\left(P\left(\phi\left(\mathbf{X}^{0}\right)\right)\right) J_{\phi} d \mathbf{X}^{0}
$$

where $\mathbf{X}^{0}$ is a point of the iso-parametric element and $J_{\phi}$ the Jacobian determinant of the transformation between the iso-parametric and deformed elements in the physical space $(c f$. Fig. 2). In other words, it means that the mesh is no longer projected onto the reference image, as it was the case before. Only the quadrature points will be projected. The positions of those points are far more precise because the elements are not artificially rebuilt from the projection of the vertices of the elements. The interest quantities are searched for in the images but the computation stay in the physical space.

Up to now, the choice of the quadrature rule $\left(\mathbf{X}_{k}\right.$ and $\left.\omega_{k}^{e}\right)$ was not precised. In FE simulation, Gauss-Legendre quadrature is used since it is known to be optimal for the integration of polynomial functions. In opposition, in DIC, this choice is not relevant, because the gradient of the image $\nabla f$ is short varying. In the following, a more simple rectangle-like quadrature rule based on evenly placed points will be used. It has the advantage to, at least, be exact for the computation of the area of an element as it is illustrated in Fig. 4 which is not the case with classical pixel integration methods (cf. Fig. 3).

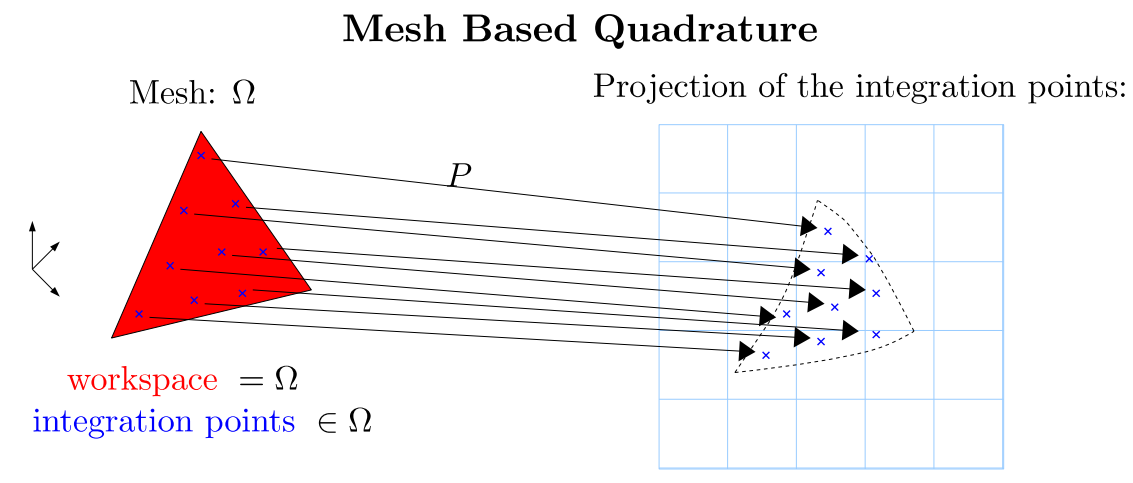

Figure 4: With a mesh based quadrature, the workspace is the domain of definition of the finite elements (the computed area, in red, is exact)

In order to use exhaustively the image data, a sufficiently large number of physical integration points have to be considered in order to have, at least, (the projection of) one integration point per pixel in the image. For that, the following procedure was adopted: each node is projected in the reference image, then the maximal number of pixels between two nodes (the diameter of the element) is determined for each element. Then, the reference element is subdivided into nested homothetic sub-triangle or quadrangles. Thus, the quadrature points $\mathbf{X}$ are the center of gravity of these sub-triangles or quadrangles and the weight coefficients $\omega_{k}^{e}$ are 
the associated areas which are constant over a given element. (cf. Fig. 5).

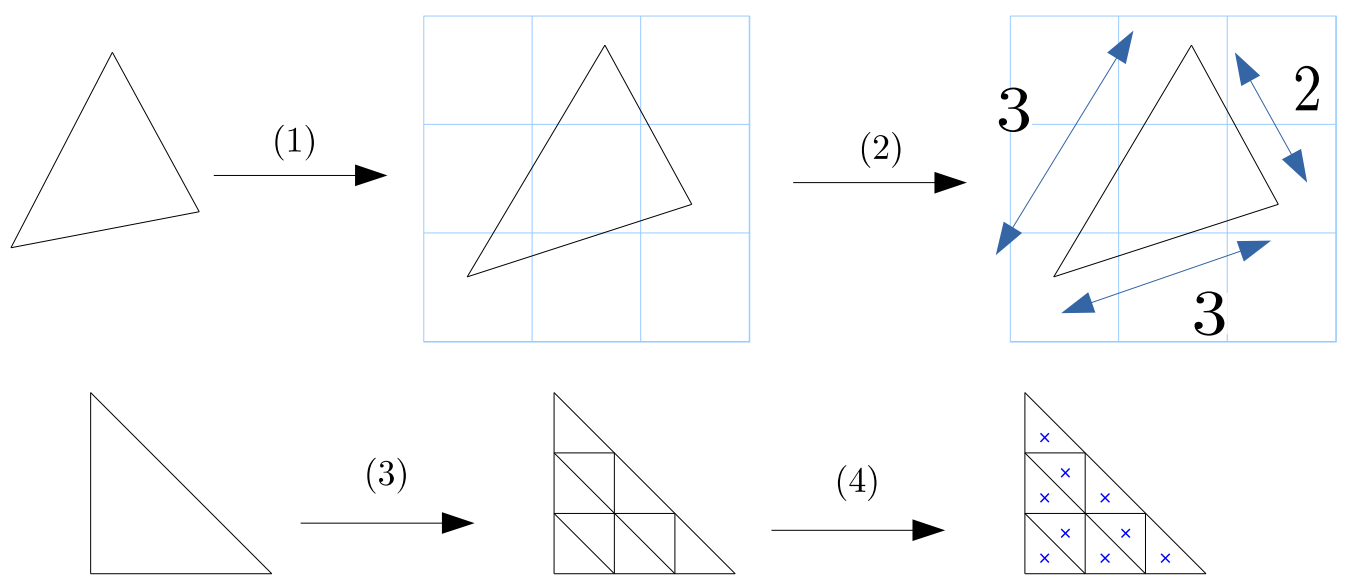

Figure 5: Subdivision of an element of an area $A$ : (1) projection of the nodes of the element on the reference images, (2) computing of the diameter $d$ of the element (here, $d=3$ pixels), (3) subdivision of the iso-parametric element by subdividing each edges with the diameter, (4) computation of the gravity center of each sub-element. It creates $d^{2}$ sub-elements of the same area $a=A / d^{2}$ in the real element. This is exactly the same for quadrangles

Remarks.

1. The positions and the number of integration points obviously depend on the mesh. If the mesh is refined, the number and the positions of the mesh could change.

2. The number of integration points (which is more or less the number of pixels for standard 2D-DIC) has a direct impact on the computational cost, in particular when using high definition sensors. In the present paper, since a front-parallel setup is considered, the number of integration points is almost the same than the number of pixels. This implies that the computational cost is merely unchanged. Nevertheless, if needed, some methods have been proposed to address the issue of numerical efficiency, such as [34, 9, 10, 35].

In the next sections, it will be shown that this choice (using at least one integration point per pixel) is a good compromise between measurement uncertainties and computation cost. This formulation in the physical space and associated quadrature rule will be referred to as Mesh Based Formulation in the following.

\section{A first perfect projector}

\subsection{Description of the projective model}

In this section, a simple linear and constant projector is considered. It is based on a frontparallel camera mode $]^{1}$ without distortions but with coincident spaces. Thus, the projector only depends on the scale factor $k$ and is written:

$$
\mathbf{x}=P(\mathbf{X})=\left(\begin{array}{c}
N_{f}-k Y \\
k X
\end{array}\right)
$$

where $N_{f}$ denotes the number of pixel of the image in the vertical direction. The scale factor $k$ corresponds to the number of pixels per meter: the scale transformation between the physical

\footnotetext{
${ }^{1}$ this model is based on a pinhole model with $\mathbf{d}=0$ and $\mathbf{T}=I_{4} \quad c f$. Sect. 5.1
} 
position and the coordinates in pixels. As explained above, the correlation operator $\mathbf{M}$ involves the spatial gradient of this projector. With such a simple model, the gradient is constant which means that the correlation matrix does not need to be rebuild at each iteration:

$$
\nabla P=\left(\begin{array}{cc}
0 & -k \\
k & 0
\end{array}\right)
$$

This simple model of projection makes the Mesh based formulation very close to the classical pixel formulation. Only the quadrature rule differs in this case. In the next section, the effect of the quadrature on measurement uncertainties is analysed.

\subsection{Effect on measurement uncertainties}

In order to compare both methods, a real image ( $c f$. Fig. 6) was taken as reference. A set of synthetic deformed state images were generated by prescribing a pure translation of magnitude 0.3, 0.4 and 0.5 pixel in the horizontal direction using image interpolations based on the shift / modulation property of the Fourier transforms.

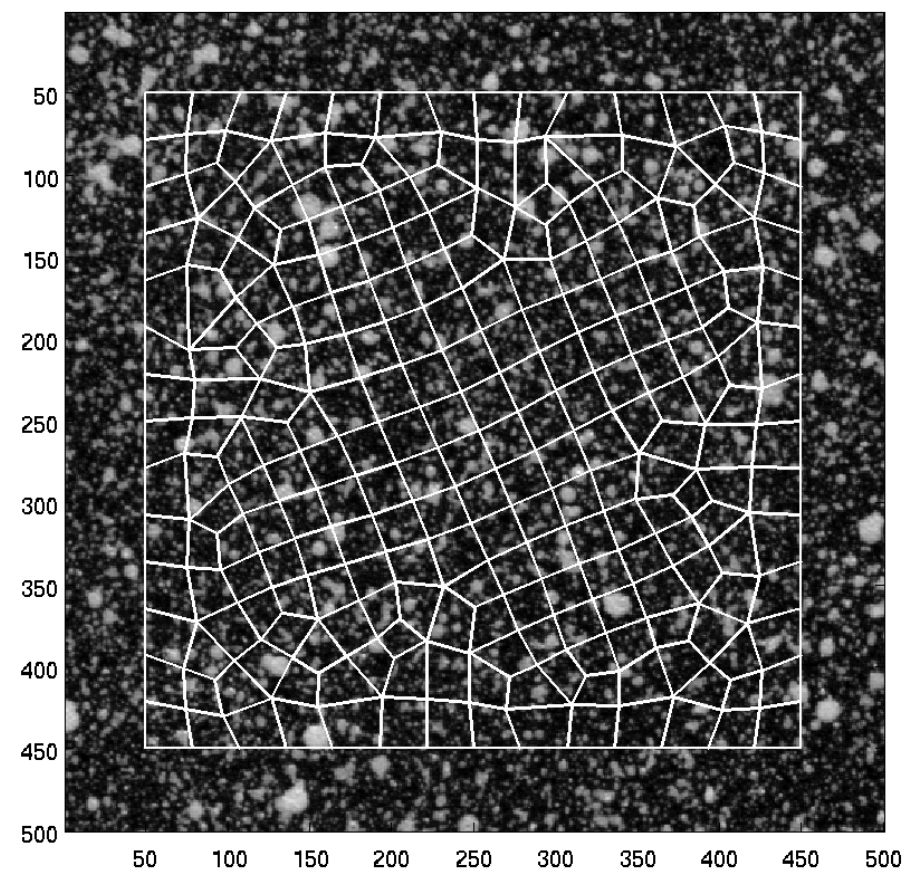

Figure 6: Reference image with an initial mesh with quadrangle (mean element size $=10$ pixels)

For this analysis, several unstructured meshes were used with different sizes of elements ranging from 4 to 30 pixels per element edges. Both linear quadrangles and triangles were used for comparison purposes. The same meshes are used to compare the quadratures rules. For each size and type of elements of the mesh, and for each deformed state images, a displacement measurement is performed. Then the standard deviation and the bias of the displacement error (defined by the difference between the prescribed and measured displacement fields) are computed and their values are averaged over the 3 shift magnitudes [15]. The results are plotted in Fig. 7. 

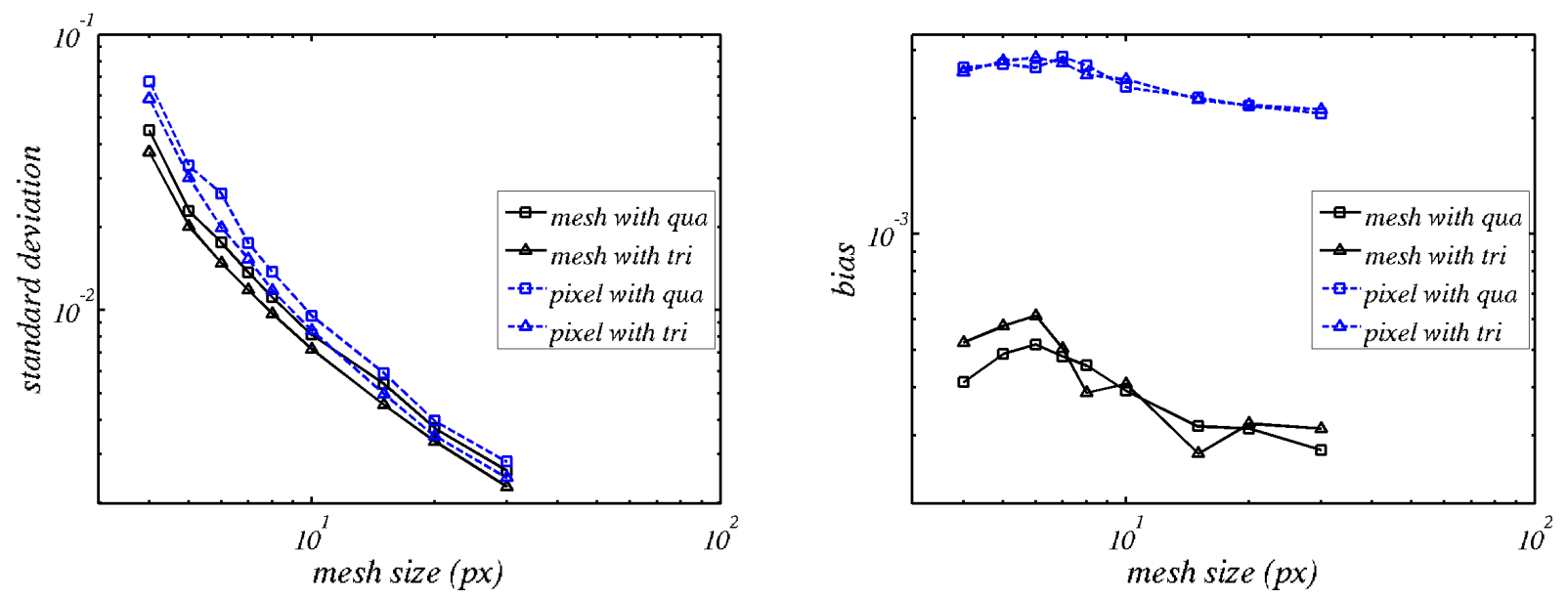

Figure 7: Evolution of the displacement standard uncertainty and the bias with respect to sub-pixel prescribed displacement for different sizes of elements. The results are computed for a Mesh Based Integration (mesh) and compared to the results of the Pixel Integration (pixel) with quadrangles (qua) or triangles (tri)

The displacement standard uncertainties are comparable even if it seems that the proposed quadrature rule gives slightly better results ( $c f$. Fig. 7). In opposition, it is clear that the pixel integration yields a systematic error which is reduced by one order of magnitude when using the proposed more accurate quadrature rule.

\subsection{Effect of the number of integration points}

Previously, the number of integration points was computed in order to have at least one point per pixel. In this section, the choice of the number of quadrature points of the proposed mesh based formulation is discussed. The mesh is fixed with an mean element size of 20 pixels.
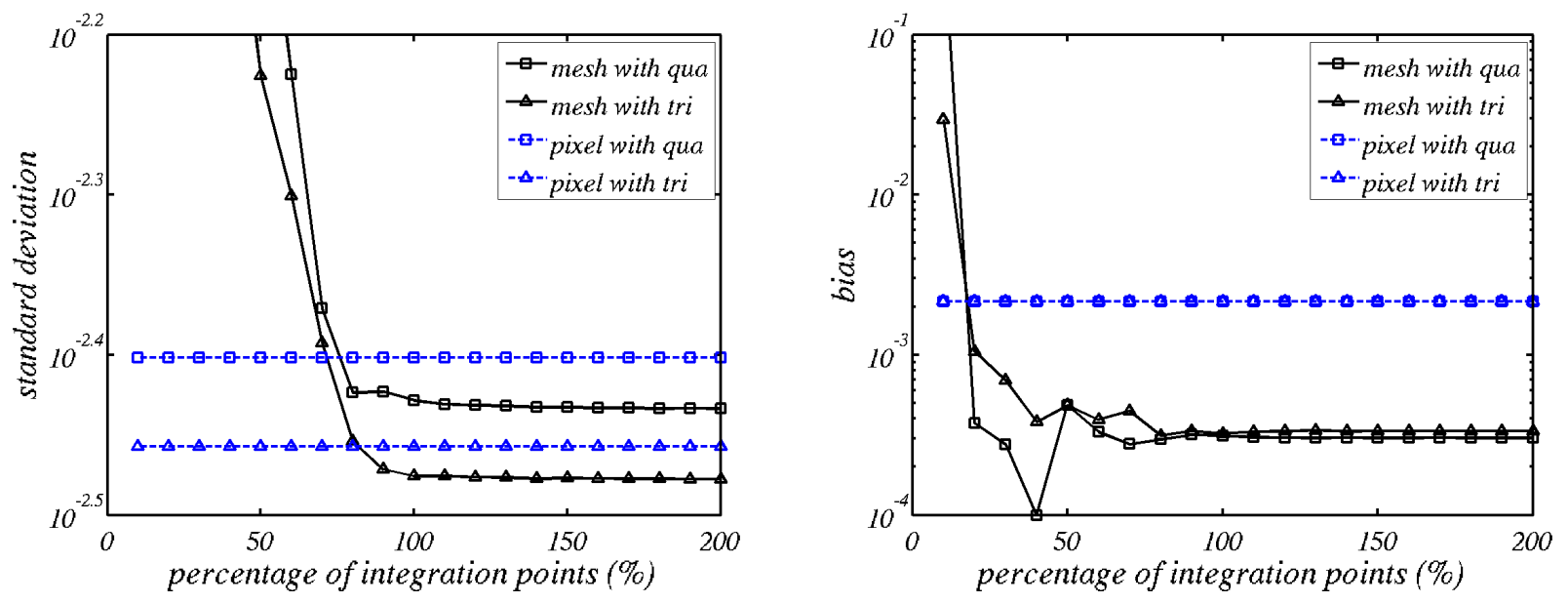

Figure 8: Evolution of the displacement standard uncertainty and bias with respect to sub-pixel prescribed displacement for different number of quadrature points (mean element size $=20$ pixels). The results are computed for a Mesh Based Integration (mesh) and compared to the results of the Pixel Integration ( pixel) with quadrangles $(q u a)$ or triangles $($ tri $)$

In Fig. 8, the effect of the number of quadrature points on the measurement uncertainties is analysed as a function of the ratio of the average number of points to the average number of 
pixels by element in percent. It can be seen that the choice proposed previously to put approximately as many integration points as the number of pixels in each element is optimal. Indeed, it allows to consider the minimum set of integration points without affecting the measurement uncertainty. Taking more points would lead to useless extra computational costs, whereas using less points would lead to higher measurement uncertainties.

\section{Application to an enhanced projector taking into account lens distortions}

One of the main advantages of using such a formulation written in the physical space is that it is possible to use a more complex camera model, for instance including the actual distortions of the lens directly during measurement. Indeed, taking into account distortion is not new to DIC [36, 37, 38, 24, 39, 40, 41, 23].

In the next section, an example of a camera model is described [42, 30, 43] and its use in the physical formulation of DIC is explained. Then, a virtual test based on synthetic images is analysed in order to estimate the benefits of including lens distortions.

\subsection{An enhanced Model of a Camera}

\section{Linear Model}

A general projective transformation is a linear transformation mapping any arbitrary physical world point $\mathbf{X}_{w}=(X, Y, Z)^{\top}$ (denoted by $\widetilde{\mathbf{X}}_{w}$ with homogeneous coordinates in the projective space $\left.\mathbb{P}^{3}\right)$ into a $2 \mathrm{D}$ point $\mathbf{x}=(u, v)^{\top}\left(\tilde{\mathbf{x}}\right.$ in the projective space $\left.\mathbb{P}^{2}\right)$. It consists of three consecutive transformations.

First, a rigid-body transformation (rotation + translation) $\mathbf{T}$ transforming a physical point $\widetilde{\mathbf{X}}_{w}$ in the world coordinate system $\mathcal{R}_{w}$ into a physical point $\widetilde{\mathbf{X}}_{c}$ in the imaging sensor coordinate system $\mathcal{R}_{c}$ :

$$
\widetilde{\mathbf{X}}_{c} \cong \underbrace{\left(\begin{array}{cc}
\mathbf{R} & \mathbf{t} \\
\mathbf{0}^{\top} & 1
\end{array}\right)}_{\mathbf{T}} \widetilde{\mathbf{X}}_{w}
$$

with $\mathbf{R}$ the rotation matrix and $\mathbf{t}$ the translation vector. The parameters of this transformation $\mathbf{T}$ are called extrinsic parameters and represent the orientation and the position of the imaging system in the world.

Second, a transformation $\mathbf{P}$ mapping a point in $\mathbb{P}^{3}$ to a point in $\mathbb{P}^{2}$. This latter transforms the physical point $\widetilde{\mathbf{X}}_{c}$ into a $2 \mathrm{D}$ point in the retinal plane $\tilde{\mathbf{x}}_{r}$ :

$$
\tilde{\mathbf{x}}_{r} \cong \underbrace{\left(\begin{array}{cccc}
\mathrm{f} & 0 & 0 & 0 \\
0 & \mathrm{f} & 0 & 0 \\
0 & 0 & 1 & 0
\end{array}\right)}_{\mathbf{P}} \widetilde{\mathbf{X}}_{c}
$$

where $f$ is the focal length.

Finally, an affine transformation $\mathbf{A}$ is applied to transform the $2 \mathrm{D}$ point $\tilde{\mathbf{x}}_{r}$ into a $2 \mathrm{D}$ point $\tilde{\mathbf{x}}$ in the distortion-free image plane:

$$
\tilde{\mathbf{x}} \cong \underbrace{\left(\begin{array}{ccc}
\alpha_{x} & \alpha_{x} \cot \theta & c_{x}+c_{y} \cot \theta \\
0 & \frac{\alpha_{y}}{\sin \theta} & \frac{c_{y}}{\sin \theta} \\
0 & 0 & 1
\end{array}\right)}_{\mathbf{A}} \tilde{\mathbf{x}}_{r}
$$


where $\alpha_{x}$ and $\alpha_{y}$ are the horizontal and vertical aspect ratio respectively. $c_{x}$ and $c_{y}$ are the horizontal and vertical coordinates of the intersection of the optical axis with the image plane respectively, also referred to as principal point. This latter point should match the center of the image. $\theta$ is the angle between the $x$-axis and the $y$-axis (cf. Fig. 10), also referred to as skew angle. Usually, the horizontal and vertical axis are almost perpendicular (i.e. $\theta \approx \frac{\pi}{2}$ ), then:

$$
\mathbf{A}=\left(\begin{array}{ccc}
\alpha_{x} & 0 & c_{x} \\
0 & \alpha_{y} & c_{y} \\
0 & 0 & 1
\end{array}\right)
$$

Note that the two last transformations (21) and (23) are almost always combined in only one transformation: AP. In order to introduce the parametric distortion model (as explain in the sequel), the latter transformation is usually replaced by a canonical projection $\Pi$ and the transformation $\mathbf{K}$ both defined as following:

$$
\begin{aligned}
\boldsymbol{\Pi} & =\left(\begin{array}{llll}
1 & 0 & 0 & 0 \\
0 & 1 & 0 & 0 \\
0 & 0 & 1 & 0
\end{array}\right) \\
\mathbf{K} & =\left(\begin{array}{ccc}
f_{x} & 0 & c_{x} \\
0 & f_{y} & c_{y} \\
0 & 0 & 1
\end{array}\right)
\end{aligned}
$$

where $f_{x}=\alpha_{x} \mathrm{f}$ and $f_{y}=\alpha_{y} \mathrm{f}$. $\mathbf{K}$ is called intrinsic matrix. Its parameters are called intrinsic parameters and represent the internal parameters of the imaging sensor. Note that the focal lens is encapsulated in $\mathbf{K}$ and not in the projection $\boldsymbol{\Pi}$.

Figure 9 summarizes the projection process from $\mathcal{R}_{w}$ to the distortions-free image plane.

\section{Projection model}

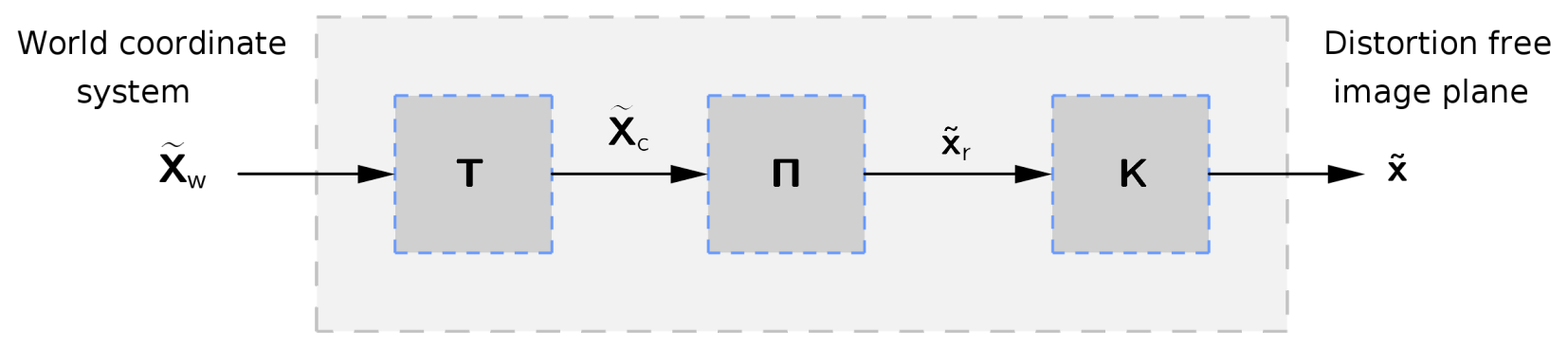

Figure 9: Diagram of the projection process from the world coordinate system to the distortions-free image plane.

Since, in this paper, only 2D-DIC is considered, all the images are taken from a front-parallel view. Consequently, $\overrightarrow{Z_{w}}$ (the z-axis of $\mathcal{R}_{w}$ ) and $\vec{Z}_{c}$ (the z-axis of $\mathcal{R}_{c}$ ) are parallel, so that $Z$ coordinates can be considered as constant. Fig. 10 summarizes the projection process in the front-parallel case.

Note that the presented projection-perspective camera model, which does not include distortions, is linear in the projective space (i.e. from $\mathbb{P}^{3}$ to $\mathbb{P}^{2}$ ). However, due to the canonical projection $\Pi$, this model is not linear in the euclidian space (i.e. from $\mathbb{R}^{3}$ to $\mathbb{R}^{2}$ ).

Lastly, a tricky point consists in the images storage. Indeed the images $f$ and $\bar{g}$ are stored 


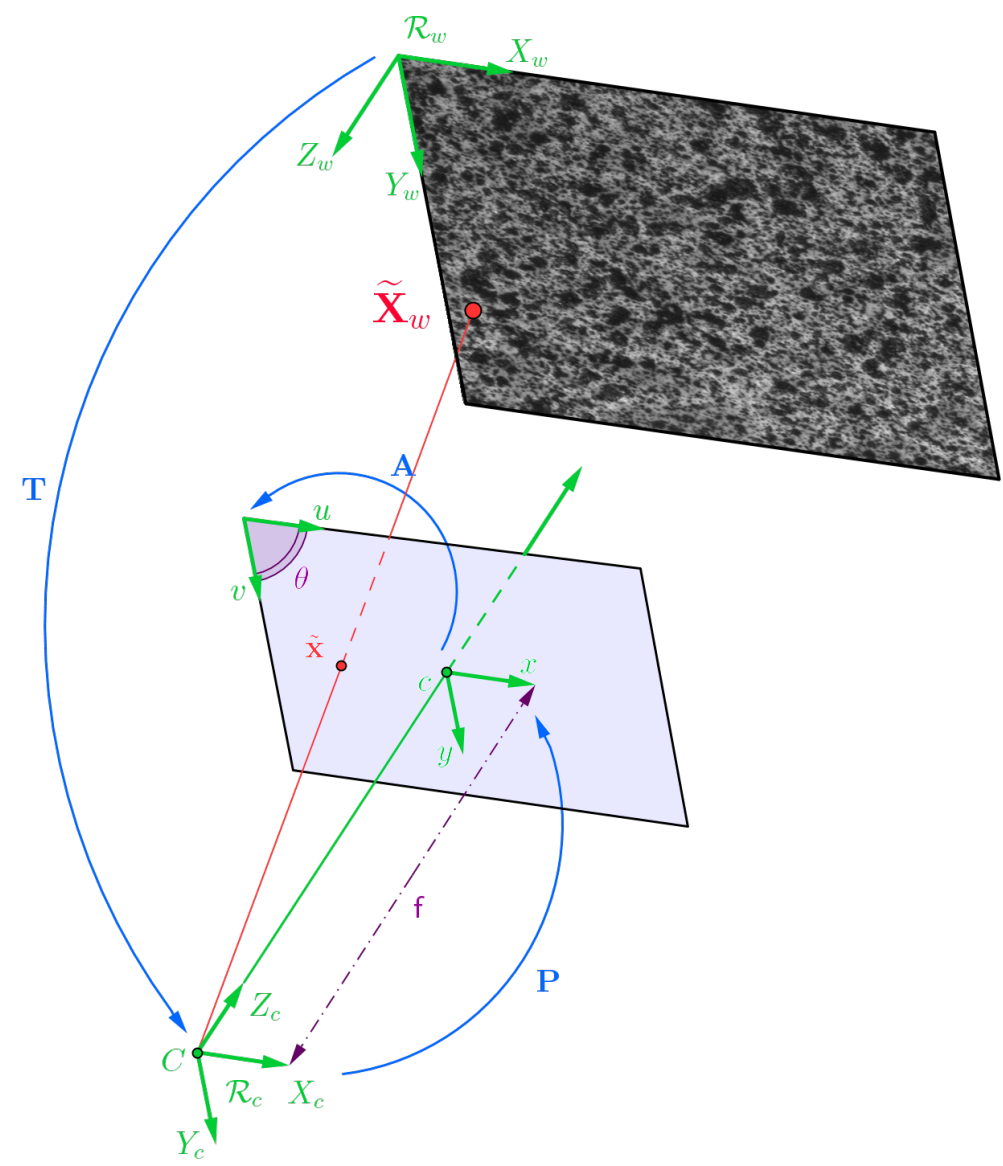

Figure 10: The three elementary transformations of the camera pin-hole model for a front-parallel view.

as tables (or matrices) of pixels $f_{i, j}$ at row $i$ and column $j$. Hence, the corresponding coordinate system (whose center is in the top left corner) requires an additional linear transformation composed of a $90^{\circ}$-rotation and a translation. This latter transformation is given by: $90^{\circ}$ rotation and translation expressed by:

$$
\left(\begin{array}{l}
u^{\prime} \\
v^{\prime}
\end{array}\right)=\left(\begin{array}{cc}
0 & -1 \\
1 & 0
\end{array}\right)\left(\begin{array}{l}
u \\
v
\end{array}\right)+\left(\begin{array}{c}
N_{f} \\
0
\end{array}\right) .
$$

In the following, different approaches for taking lens distortions into account are presented.

\section{Distortion Models}

Distortion models can be classified in two categories: the parametric models and the nonparametric models. Despite their different approaches, the key point consists in their different locations in the global projection diagram of Fig. 9.

In the parametric model, denoted by $\mathrm{D}$, only three types of distortions are considered: the radial distortion $\Delta_{r}$ (par-axial approximation), the decentralize distortion $\Delta_{d}$ (imperfect alignment of the optical centres of all lens) and the prismatic distortion $\Delta_{p}$ (bad parallelism of the lens between them and/or with the plane of the detector). For more advanced informations, the reader can refer to [42, 44, 45]. The expression of $\mathrm{D}, \Delta_{r}, \Delta_{d}$ and $\Delta_{p}$ are given by:

$$
\begin{aligned}
& \mathrm{D}: \mathbb{R}^{2} \\
& \tilde{\mathbf{x}}_{r} \mapsto \mathbb{R}^{2} \\
& \tilde{\mathbf{x}}_{d}=\tilde{\mathbf{x}}_{c}+\Delta_{r}\left(\tilde{\mathbf{x}}_{c}\right)+\Delta_{d}\left(\tilde{\mathbf{x}}_{c}\right)+\Delta_{p}\left(\tilde{\mathbf{x}}_{c}\right)
\end{aligned}
$$




$$
\begin{gathered}
\Delta_{r}\left(\tilde{\mathbf{x}}_{c}\right)=\left(\begin{array}{c}
x \sum_{i=1}^{3} \kappa_{i}\left(x_{c}^{2}+y_{c}^{2}\right)^{i} \\
y \sum_{i=1}^{3} \kappa_{i}\left(x_{c}^{2}+y_{c}^{2}\right)^{i}
\end{array}\right) \\
\Delta_{d}\left(\tilde{\mathbf{x}}_{c}\right)=\left(\begin{array}{l}
2 d_{1} x_{c} y_{c}+d_{2}\left(3 x_{c}^{2}+y_{c}^{2}\right) \\
2 d_{1} x_{c} y_{c}+d_{2}\left(x_{c}^{2}+3 y_{c}^{2}\right)
\end{array}\right) \\
\Delta_{p}\left(\tilde{\mathbf{x}}_{c}\right)=\left(\begin{array}{c}
p_{1}\left(x_{c}^{2}+y_{c}^{2}\right) \\
p_{2}\left(x_{c}^{2}+y_{c}^{2}\right)
\end{array}\right)
\end{gathered}
$$

In this approach [46, 47, 48, lens distortions are considered between $\mathbf{\Pi}$ and $\mathbf{K}$ (cf. Fig. 11). More precisely, lens distortions are introduced after the projection on the retinal plane $\mathbf{x}_{r}=$ $\left(x_{r}, y_{r}\right)^{\top}$ and before applying the intrinsic parameters. Figure 11 summarizes the introduction of the parametric model into the projection diagram.

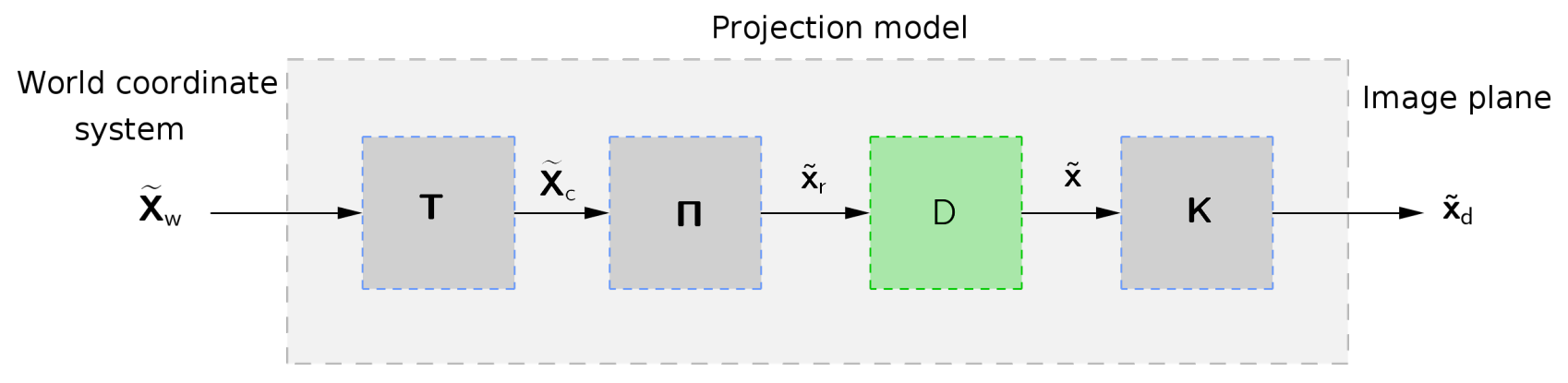

Figure 11: Diagram of the projection process from the world coordinate system to the distortions image plane in the parametric case.

Due to the good quality of the modern lenses, a development at the $3^{\text {rd }}$ order of the radial distortion $\Delta_{d}$ is enough for most of the camera lens with a focal length greater than $5 \mathrm{~mm}$.

In the non-parametric model [49], the main idea is to recover the distortion shape from the remaining reprojection errors of the calibration of a pure projective model and fitting it thanks to an interpolation method (e.g. spline interpolation). This approach allows to represent any type of distortion field, even complex. However, this model, denoted by I, is included before applying the intrinsic matrix $\mathbf{K}$ ( $c f$. Fig. 12).

Moreover, for the sakes of simplify and computational efficiency of the distortion removal process, the interpolation I can be replaced by its reciprocal $\mathrm{I}^{-1}$, the distortion correction $(c f$. Fig. 13.

Choosing a distortion model is strongly related to the informations available. Indeed, if no calibration informations are available or in case of complex lens, a non-parametric model can be considered. However, if the calibration parameters are available, the parametric model will be more accurate.

\subsection{Synthetic test case}

In this section, the aforementioned non-linear projector is used in the mesh based DIC method described in Sect. 3 for the analysis of a synthetic test case. Like this, it is possible to 
Projection model

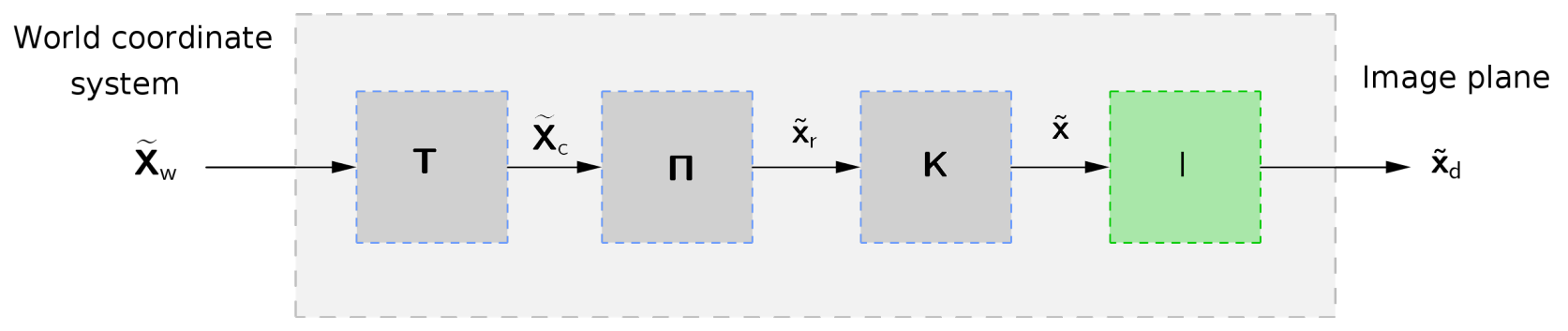

Figure 12: Diagram of the projection process from the world coordinate system to the distortions image plane in the non-parametric case (direct interpolation).

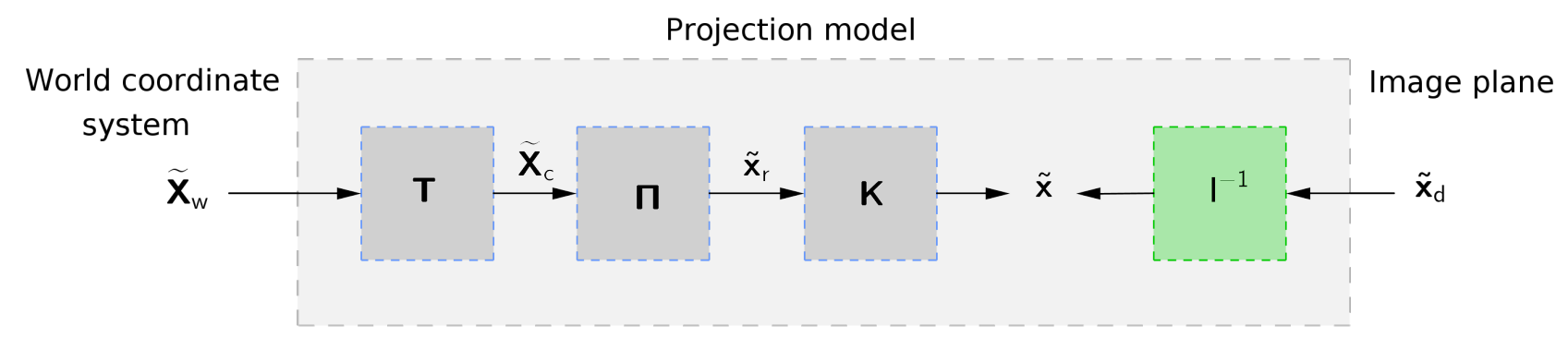

Figure 13: Diagram of the projection process from the world coordinate system to the distortions image plane in the non-parametric case (inverse interpolation).

filter the effect of lens distortions during the measurement.

In practice, a real physical experimentation is performed in order to feed the non-linear projector with realistic intrinsic parameter values. For that, the configuration is as followed: a CCD camera (Pike F-145b, 8 bit, 1388×1036 px) equipped with a Nikon Nikkor $50 \mathrm{~mm}$ f2 aperture 11 lens was set up, allowing a field-of-view of about $270 \times 200 \mathrm{~mm}^{2}$ and an optical resolution roughly equal to $5 \mathrm{px} / \mathrm{mm}$. Then 110 images of a conventional target were taken. The calibration (via a projection-perspective first-order radial model sufficient for this kind of lens) was done using the commercial software Vic-3 $3 \mathrm{D}^{\mathrm{TM}}$ from Correlated Solutions, Inc. [] for reliable results. Intrinsics were: $u_{0}=706.053 \mathrm{px}, v_{0}=503.725, k_{u}=k_{v}=8381.24 \mathrm{px}$ and $r_{1}=-0.0321253$ with a reprojection error of $0.05 \mathrm{px}$. Note in addition that a second camera was also positioned (stereo pan angle of almost $15^{\circ}$ ) and the results from a stereo-rig calibration are close to those from a single camera calibration.

Then a set of synthetic images is created. It has to be noted that the synthetic images are created using the real distortion parameters that were previously characterised experimentally. The only way to quantify the efficiency of the proposed method in reducing the measurement uncertainty over a standard DIC, is to develop a synthetic test case as classically done in the literature $([50,51])$ in order to have a ground truth reference. Otherwise, the reference becomes unknown and it would be difficult to conclude. First a realistic synthetic speckle pattern is mapped onto a plane in the physical space, such that a gray level $F_{p}(\mathbf{X})$ is associated to each physical point $\mathbf{X}$. Then, for any integer pixel position in the reference image plane $\mathbf{x}_{p}$, the coordinates $\mathbf{X}_{p}$ of the corresponding point in the physical space are obtained by solving the non linear equation $\mathbf{x}_{p}-P\left(\mathbf{X}_{p}\right)=0$, thanks to a Newton algorithm. Then a reference image $f$ is synthesised by estimating the gray level $F_{p}\left(\mathbf{X}_{p}\right)$ associated to the physical point $\mathbf{X}_{p}$ for each integer pixel position $\mathbf{x}_{p}$. Finally a deformed state image $\bar{g}$ is synthesised by estimating the 
gray level $F_{p}\left(\mathbf{X}_{p}+\mathbf{S}\right)$ where $\mathbf{S}$ is a rigid body shift in the physical space.

In the following, a rather large shift $\mathbf{S}=(400,200,0)$ pixels is chosen in order to highlight the effect of distortions. Then a Pixel and a Mesh Based DIC between the distorted images $f$ and $\bar{g}$ were performed with different element types and sizes. In addition, the effect of the number of quadrature points on the measurement uncertainties is again analysed as a function of the ratio of the average number of points to the average number of pixels by element in percent. Like previously, the measurement uncertainties are quantified as the standard deviation and the bias (cf. Fig. 14, 15).
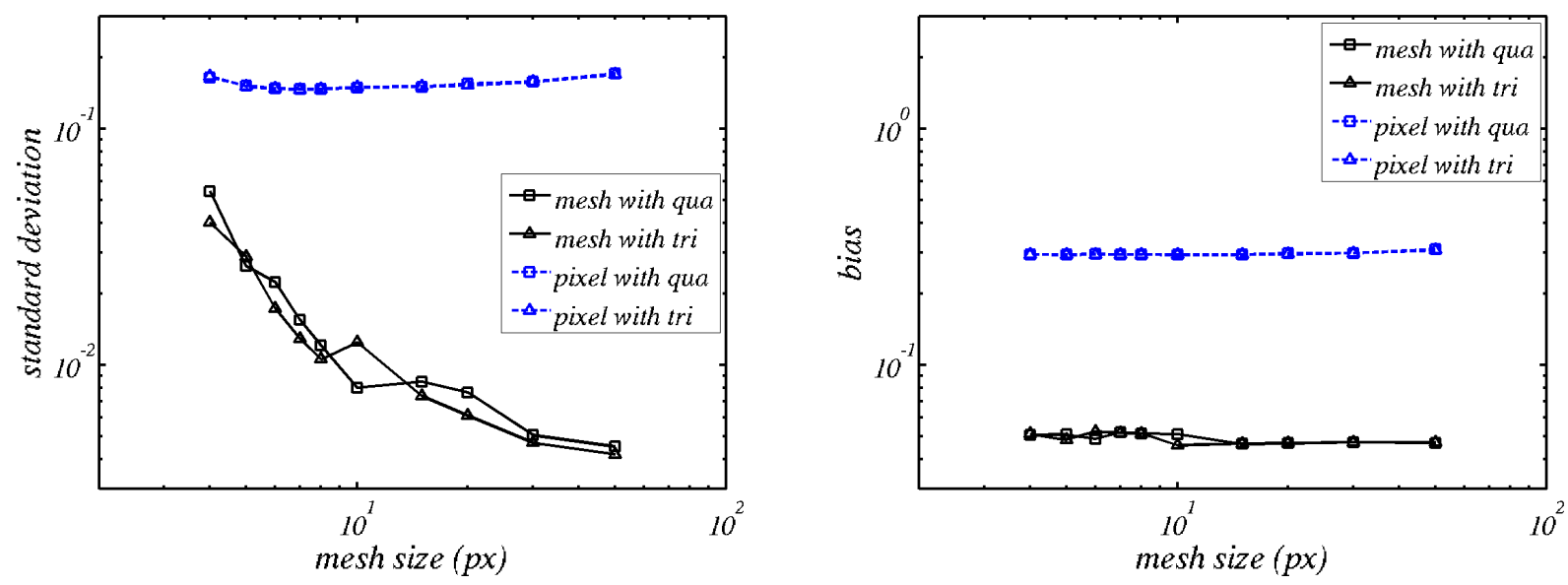

Figure 14: Evolution of the displacement standard uncertainty and the bias with respect to sub-pixel prescribed displacement for different elements sizes on a distorted image. The results are computed for a Mesh Based Integration (mesh) and compared to the results of the Pixel Integration (pixel) with quadrangles (qua) or triangles $($ tri $)$
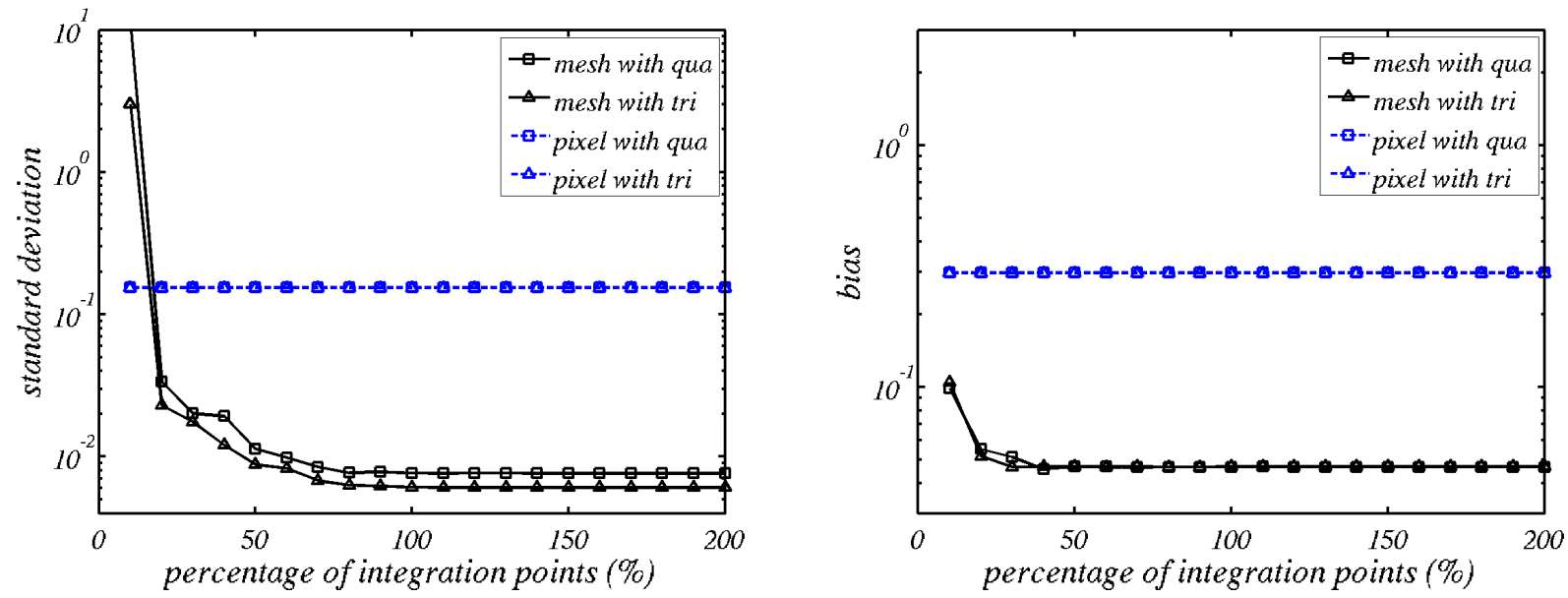

Figure 15: Evolution of the displacement standard uncertainty and bias with respect to sub-pixel prescribed displacement for different number of quadrature points on a distorted image (mean element size $=20$ pixels). The results are computed for a Mesh Based Integration (mesh) and compared to the results of the Pixel Integration (pixel) with quadrangles (qua) or triangles (tri)

This was done in order to see that one integration point per pixel is the minimum, and that it is useless to have more information. As expected, all curves from Pixel Integration are widely above those from Mesh Based Integration because in the former case, lens distortions are not 
taking into account which is crippling for such large displacements. Consequently, effects of the elements size ( $c f$. Fig. 14) or of the number of integration points ( $c f$. Fig. 15) have become of second order in the errors.

It is interesting to see the displacement field measured on a square field of view of $300 \times$ $300 p x^{2}$ for a given element size with both methods ( $c f$. Fig. 16). With a classical Pixel Integration, the intrinsic parameters of the camera are not taken into account, which means that the measured displacement is not uniform and the iso-distortion circles can be seen on the displacement field.
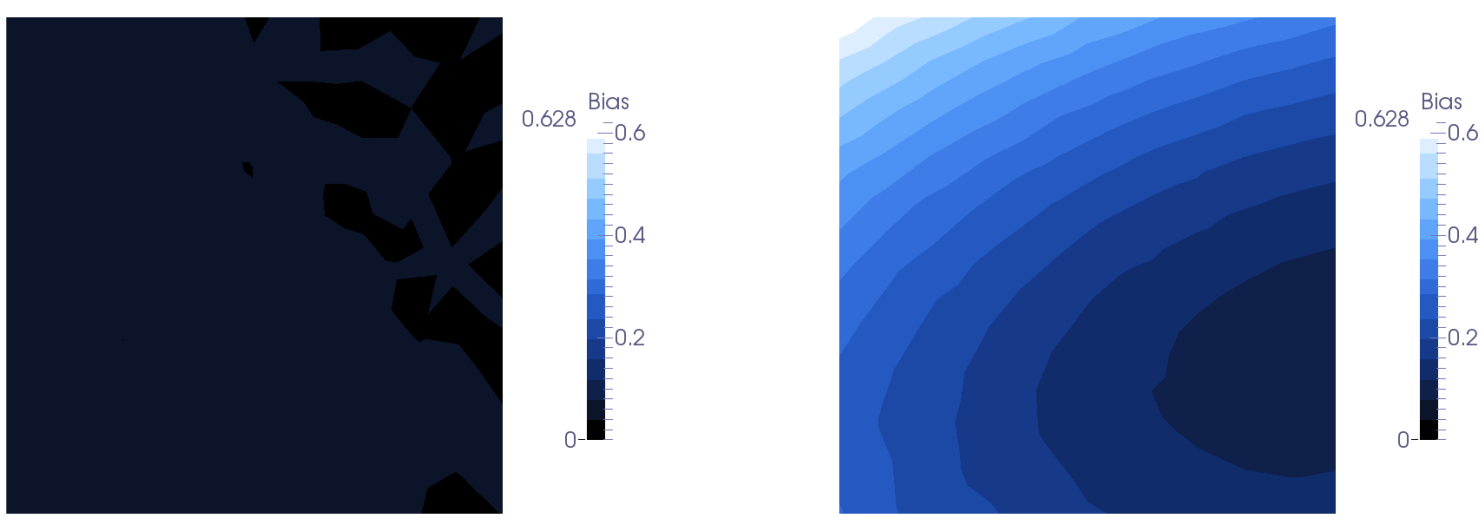

Figure 16: Exact error of the vertical component of the displacement field in pixels measured on distorted images with the Mesh Based Integration (left) with a calibrated camera or with a classical Pixel Integration (right) without taking account the camera's intrinsic parameters.

Thus, the Mesh Based Integration becomes clearly more precise especially if large displacements are considered.

\section{Conclusion}

In a classical 2D FE-DIC method, the integration of the gray-level difference is still closely related to the pixels of the reference image. Not only the $R O I$ is an integer set of pixels (the element shapes are approximated), but the quadrature rule also uses pixels $\mathbf{x}$ as integration points and their area as weights.

In opposition, in this paper, it is chosen to minimise the gray-level difference in the physical space with a dedicated quadrature method. The region of interest is defined in the physical space by the mesh geometry. The quadrature rule is based on points $\mathbf{X}$ in the world coordinate system. A projective model is used to compute the corresponding gray-levels in both reference $f(P(\mathbf{X}))$ and deformed $\bar{g}(P(\mathbf{X}))$ images. The number of quadrature points $\mathbf{X}$ is set such that in any pixel, one can find at least the projection $P(\mathbf{X})$ of one point. It has to be noted that, with this method, the measured displacement is then directly expressed in the physical space.

At first, thanks to the proposed quadrature technique, it is shown that a systematic measurement error is avoided, even for a simple projection model (without lens distortions). Then, an enhanced camera model is used as a projector. In practice, a pin-hole model is implemented in order to consider lens distortions. Even if the latter have much less impact when dealing with 
small strains, it is shown that they have to be considered when measuring large displacements. Distortions should be taken into account for displacement-based FEMU approaches. The proposed method should allow performing 2D FE-DIC measurements in tricky optical setups, for example when using microscopes [52], wide-angle lenses or portholes.

With the computed physical displacement, it becomes easier to use a displacement-based FEMU method or to perform a 2D identification [53]. Obviously, the proposed formulation will have even a greater interest in the context of Finite Element Stereo (or Multiview) Digital Image Correlation for the measurement of 3D surface displacement.

\section{Acknowledgments}

This work was funded by the French "Agence Nationale de la Recherche" under the grant ANR-12-RMNP-0001 (VERTEX project).

\section{References}

\section{References}

[1] B. D. Lucas, T. Kanade, An iterative image registration technique with an application to stereo vision, in: Proceedings of Imaging Understanding Workshop, 1981, pp. 121-130. URL http://www .robots.ox.ac.uk/ cvrg/trinity2003/lucas81-2.ps

[2] M. A. Sutton, W. J. Wolters, W. H. Peters, W. F. Ranson, S. R. McNeill, Determination of displacements using an improved digital correlation method, Image and Vision Computing 1 (3) (1983) 133-139. doi : 10.1016/0262-8856(83)90064-1. URL http://www.sciencedirect.com/science/article/pii/0262885683900641

[3] M. A. Sutton, S. R. McNeill, J. D. Helm, M. L. Boone, Measurement of Crack Tip Opening Displacement and Full-field Deformations During Fracture of Aerospace Materials Using 2d and 3d Image Correlation Methods, in: A. Lagarde (Ed.), IUTAM Symposium on Advanced Optical Methods and Applications in Solid Mechanics, no. 82 in Solid Mechanics and its Applications, Springer Netherlands, 2000, pp. 571-580.

URL http://link .springer .com/chapter/10.1007/0-306-46948-0_57

[4] S. Roux, F. Hild, Y. Berthaud, Correlation image velocimetry: a spectral approach, Applied Optics 41 (1) (2002) 108-115. doi:10.1364/A0.41.000108.

URL http://ao.osa.org/abstract.cfm?URI=ao-41-1-108

[5] Y. Sun, J. H. L. Pang, C. K. Wong, F. Su, Finite element formulation for a digital image correlation method, Applied Optics 44 (34) (2005) 7357-7363. doi:10.1364/A0.44. 007357.

URL http://ao.osa.org/abstract.cfm?URI=ao-44-34-7357

[6] G. Besnard, F. Hild, S. Roux, "Finite-Element" Displacement Fields Analysis from Digital Images: Application to Portevin-Le Châtelier Bands, Experimental Mechanics 46 (6) (2006) 789-803. doi:10.1007/s11340-006-9824-8. URL http://link.springer .com/10.1007/s11340-006-9824-8 
[7] F. Hild, S. Roux, R. Gras, N. Guerrero, M. E. Marante, J. Flórez-López, Displacement measurement technique for beam kinematics, Optics and Lasers in Engineering 47 (3-4) (2009) 495-503. doi:10.1016/j.optlaseng.2008.03.006.

URL http://linkinghub.elsevier.com/retrieve/pii/S0143816608000432

[8] J. Réthoré, T. Elguedj, P. Simon, M. Coret, On the Use of NURBS Functions for Displacement Derivatives Measurement by Digital Image Correlation, Experimental Mechanics 50 (7) (2010) 1099-1116. doi:10.1007/s11340-009-9304-z. URL http://link. springer .com/10.1007/s11340-009-9304-z

[9] J.-C. Passieux, J.-N. Périé, High resolution digital image correlation using proper generalized decomposition: PGD-DIC, International Journal for Numerical Methods in Engineering 92 (6) (2012) 531-550. doi:10.1002/nme.4349. URL http://onlinelibrary.wiley.com/doi/10.1002/nme.4349/abstract

[10] J.-C. Passieux, P. Navarro, J.-N. Périé, S. Marguet, J.-F. Ferrero, A Digital Image Correlation Method For Tracking Planar Motions Of Rigid Spheres: Application To Medium Velocity Impacts, Experimental Mechanics 54 (8) (2014) 1453-1466. doi: 10.1007/s11340-014-9930-y. URL http://link.springer.com/article/10.1007/s11340-014-9930-y

[11] F. Hild, S. Roux, Comparison of Local and Global Approaches to Digital Image Correlation, Experimental Mechanics 52 (9) (2012) 1503-1519. doi:10.1007/ s11340-012-9603-7. URL http://link.springer.com/article/10.1007/s11340-012-9603-7

[12] K. T. Kavanagh, R. W. Clough, Finite element applications in the characterization of elastic solids, International Journal of Solids and Structures 7 (1) (1971) 11-23. doi: 10.1016/0020-7683(71)90015-1. URL http://www.sciencedirect.com/science/article/pii/0020768371900151

[13] H. Leclerc, J.-N. Périé, S. Roux, F. Hild, Integrated Digital Image Correlation for the Identification of Mechanical Properties, in: A. Gagalowicz, W. Philips (Eds.), Computer Vision/Computer Graphics CollaborationTechniques, no. 5496 in Lecture Notes in Computer Science, Springer Berlin Heidelberg, 2009, pp. 161-171.

URL http://link .springer.com/chapter/10.1007/978-3-642-01811-4_15

[14] R. Gras, H. Leclerc, S. Roux, S. Otin, J. Schneider, J.-N. Périé, Identification of the Outof-Plane Shear Modulus of a 3d Woven Composite, Experimental Mechanics 53 (5) (2012) 719-730. doi:10.1007/s11340-012-9683-4.

URL http://link.springer.com/article/10.1007/s11340-012-9683-4

[15] J.-C. Passieux, F. Bugarin, C. David, J.-N. Périé, L. Robert, Multiscale Displacement Field Measurement Using Digital Image Correlation: Application to the Identification of Elastic Properties, Experimental Mechanics 55 (1) (2015) 121-137. doi:10.1007/ s11340-014-9872-4. URL http://link.springer.com/article/10.1007/s11340-014-9872-4

[16] F. Hild, S. Roux, Digital Image Correlation: from Displacement Measurement to Identification of Elastic Properties - a Review, Strain 42 (2) (2006) 69-80. 
doi:10.1111/j.1475-1305.2006.00258.x.

URL http://onlinelibrary.wiley.com/doi/10.1111/j.1475-1305.2006.00258.x/ abstract

[17] J. Fehrenbach, M. Masmoudi, A fast algorithm for image registration, Comptes Rendus Mathematique 346 (9-10) (2008) 593-598. doi:10.1016/j.crma.2008.03.019.

URL http://www.sciencedirect.com/science/article/pii/S1631073X08001015

[18] J. Réthoré, Muhibullah, T. Elguedj, M. Coret, P. Chaudet, A. Combescure, Robust identification of elasto-plastic constitutive law parameters from digital images using $3 \mathrm{~d}$ kinematics, International Journal of Solids and Structures 50 (1) (2013) 73-85. doi: 10.1016/j.ijsolstr.2012.09.002.

URL http://wWw.sciencedirect.com/science/article/pii/S0020768312003836

[19] A. Baldi, L. Francesconi, A. Medda, F. Bertolino, Comparing Two Damage Models Under Shear Stress, Experimental Mechanics 53 (7) (2013) 1105-1116. doi:10.1007/ s11340-013-9715-8.

URL http://link.springer.com/10.1007/s11340-013-9715-8

[20] J. v. Beeck, J. Neggers, P. J. G. Schreurs, J. P. M. Hoefnagels, M. G. D. Geers, Quantification of Three-Dimensional Surface Deformation using Global Digital Image Correlation, Experimental Mechanics 54 (4) (2014) 557-570. doi:10.1007/s11340-013-9799-1.

URL http://link.springer .com/article/10.1007/s11340-013-9799-1

[21] L. Wittevrongel, P. Lava, S. V. Lomov, D. Debruyne, A Self Adaptive Global Digital Image Correlation Algorithm, Experimental Mechanics (2014) 1-18doi:10.1007/ s11340-014-9946-3.

URL http://link.springer.com/article/10.1007/s11340-014-9946-3

[22] B. Beaubier, J.-E. Dufour, F. Hild, S. Roux, S. Lavernhe, K. Lavernhe-Taillard, CADbased calibration and shape measurement with stereoDIC: Principle and application on test and industrial parts, Experimental Mechanics 54 (3) (2014) 329-341. doi:10.1007/ s11340-013-9794-6.

URL http://link . springer .com/10.1007/s11340-013-9794-6

[23] P. Lava, W. Van Paepegem, S. Coppieters, I. De Baere, Y. Wang, D. Debruyne, Impact of lens distortions on strain measurements obtained with 2d digital image correlation, Optics and Lasers in Engineering 51 (5) (2013) 576-584. doi:10.1016/j.optlaseng.2012.12. 009.

URL http://linkinghub.elsevier.com/retrieve/pii/S0143816612003478

[24] S. Yoneyama, H. Kikuta, A. Kitagawa, K. Kitamura, Lens distortion correction for digital image correlation by measuring rigid body displacement, Optical Engineering 45 (2) (2006) 023602-023602-9. doi:10.1117/1.2168411.

URL http://dx.doi .org/10.1117/1.2168411

[25] M. A. Sutton, J. H. Yan, V. Tiwari, H. W. Schreier, J. J. Orteu, The effect of out-ofplane motion on 2d and 3d digital image correlation measurements, Optics and Lasers in Engineering 46 (10) (2008) 746-757. doi:10.1016/j.optlaseng.2008.05.005. URL http://www.sciencedirect.com/science/article/pii/S0143816608000985 
[26] B. Pan, L. Yu, D. Wu, High-Accuracy 2d Digital Image Correlation Measurements with Bilateral Telecentric Lenses: Error Analysis and Experimental Verification, Experimental Mechanics 53 (9) (2013) 1719-1733. doi:10.1007/s11340-013-9774-x. URL http://link . springer . com/10.1007/s11340-013-9774-x

[27] B. Pan, L. Yu, D. Wu, High-accuracy 2d digital image correlation measurements using lowcost imaging lenses: implementation of a generalized compensation method, Measurement Science and Technology 25 (2) (2014) 025001. doi:10.1088/0957-0233/25/2/025001. URL http://iopscience.iop.org/0957-0233/25/2/025001

[28] J. Réthoré, F. Morestin, L. Lafarge, P. Valverde, 3d displacement measurements using a single camera, Optics and Lasers in Engineering 57 (2014) 20-27. doi:10.1016/j. optlaseng.2014.01.009. URL http://www.sciencedirect.com/science/article/pii/S0143816614000104

[29] S. Chambon, A. Crouzil, Dense matching using correlation: new measures that are robust near occlusions., in: BMVC, 2003, pp. 1-10. URL http://www.researchgate.net/profile/Sylvie_Chambon/publication/ 228771410_Dense_matching_using_correlation_new_measures_that_are_robust_ near_occlusions/links/0c9605350eaa0d68eb000000.pdf

[30] M. A. Sutton, J. J. Orteu, H. W. Schreier, Image Correlation for Shape, Motion and Deformation Measurements - Basic Concepts, Theory and Applications, Springer, 2009. URL http://www . springer . com/materials/mechanics/book/978-0-387-78746-6

[31] B. Pan, H. Xie, Z. Wang, Equivalence of digital image correlation criteria for pattern matching, Applied optics 49 (28) (2010) 5501-5509. URL http://www . osapublishing.org/abstract.cfm?uri=ao-49-28-5501

[32] R. Fedele, L. Galantucci, A. Ciani, Global 2d digital image correlation for motion estimation in a finite element framework: a variational formulation and a regularized, pyramidal, multi-grid implementation, International Journal for Numerical Methods in Engineering 96 (12) (2013) 739-762. doi:10.1002/nme.4577. URL http://onlinelibrary.wiley.com/doi/10.1002/nme.4577/abstract

[33] O. C. Zienkiewicz, R. L. Taylor, P. Nithiarasu, J. Z. Zhu, The finite element method, Elsevier/Butterworth-Heinemann, Oxford; New York, 2005.

[34] F. Champagnat, A. Plyer, G. L. Besnerais, B. Leclaire, S. Davoust, Y. L. Sant, Fast and accurate PIV computation using highly parallel iterative correlation maximization, Experiments in Fluids 50 (4) (2011) 1169-1182. doi:10.1007/s00348-011-1054-x. URL http://link . springer . com/article/10.1007/s00348-011-1054-x

[35] J.-C. Passieux, J.-N. Périé, M. Salaün, A dual domain decomposition method for finite element digital image correlation, International Journal for Numerical Methods in Engineering 102 (10) (2015) 1670-1682. doi:10.1002/nme.4868.

URL http://onlinelibrary.wiley.com/doi/10.1002/nme.4868/abstract

[36] D. Zhang, M. Luo, D. D. Arola, Displacement/strain measurements using an optical microscope and digital image correlation, Optical Engineering 45 (3) (2006) 033605-033605-9. 
doi:10.1117/1.2182108.

URL http://dx.doi .org/10.1117/1.2182108

[37] M. A. Sutton, N. Li, D. Garcia, N. Cornille, J. J. Orteu, S. R. McNeill, H. W. Schreier, $\mathrm{X}$. Li, Metrology in a scanning electron microscope: theoretical developments and experimental validation, Measurement Science and Technology 17 (10) (2006) 2613-2622. doi:10.1088/0957-0233/17/10/012. URL http://stacks.iop.org/0957-0233/17/i=10/a=012?key=crossref . 054d5d812ecb6cd6affa82aaee44f277

[38] Y. Sun, J. H. L. Pang, AFM image reconstruction for deformation measurements by digital image correlation, Nanotechnology 17 (4) (2006) 933-939. doi:10.1088/0957-4484/17/4/016.

URL http://stacks.iop.org/0957-4484/17/i=4/a=016?key=crossref . 8bc6f0bb2e7a7cb43bd09dcc03e5bcd2

[39] S. Yoneyama, A. Kitagawa, K. Kitamura, H. Kikuta, In-plane displacement measurement using digital image correlation with lens distortion correction, JSME International Journal 49 (3). doi:http://dx.doi.org/10.1299/jsmea.49.458.

[40] M. A. Sutton, N. Li, D. C. Joy, A. P. Reynolds, X. Li, Scanning Electron Microscopy for Quantitative Small and Large Deformation Measurements Part I: SEM Imaging at Magnifications from 200 to 10,000, Experimental Mechanics 47 (6) (2007) 775-787. doi: $10.1007 / \mathrm{s} 11340-007-9042-\mathrm{z}$. URL http://link.springer.com/10.1007/s11340-007-9042-z

[41] M. A. Sutton, N. Li, D. Garcia, N. Cornille, J. J. Orteu, S. R. McNeill, H. W. Schreier, X. Li, A. P. Reynolds, Scanning Electron Microscopy for Quantitative Small and Large Deformation Measurements Part II: Experimental Validation for Magnifications from 200 to 10,000, Experimental Mechanics 47 (6) (2007) 789-804. doi:10.1007/ s11340-007-9041-0.

URL http://link. springer.com/10.1007/s11340-007-9041-0

[42] D. Garcia, Mesure de formes et de champs de déplacements tridimensionnels par stéréocorrélation d'images, Ph.D. thesis, Institut National Polytechnique de Toulouse - INPT (Dec. 2001).

URL http://tel.archives-ouvertes.fr/tel-00166476

[43] R. Hartley, A. Zisserman, Multiple view geometry in computer vision, Cambridge University Press, Cambridge, UK; New York, 2003.

URL http://dx.doi .org/10.1017/CB09780511811685

[44] G. Besnard, Caractérisation et quantification de surfaces par stéréocorrélation pour des essais mécaniques du quasi statique à la dynamique ultra-rapide, Ph.D. thesis, Ecole normale supérieure de Cachan - ENS Cachan (Mar. 2010).

URL http://tel.archives-ouvertes.fr/tel-00579260

[45] F. Bugarin, Vision 3d multi-images: contribution à l'obtention de solutions globales par optimisation polynomiale et théorie des moments, Ph.D. thesis, Université de Toulouse, Institut National Polytechnique de Toulouse (INP Toulouse) (2012).

URL http://oatao .univ-toulouse.fr/8834/ 
[46] A. Fitzgibbon, Simultaneous linear estimation of multiple view geometry and lens distortion, in: Proceedings of the 2001 IEEE Computer Society Conference on Computer Vision and Pattern Recognition, 2001. CVPR 2001, Vol. 1, 2001, pp. I-125-I-132 vol.1. doi:10.1109/CVPR.2001.990465.

[47] J. H. Brito, C. Zach, K. Köser, M. J. Ferreira, M. Pollefeys, One-sided Radial Fundamental Matrix Estimation.

URL http://cvg.ethz.ch/research/distortion-in-multiple-view-geometry/ jbritoBMVC2012.pdf

[48] J.-E. Dufour, F. Hild, S. Roux, Integrated digital image correlation for the evaluation and correction of optical distortions, Optics and Lasers in Engineering 56 (2014) 121-133. doi:10.1016/j.optlaseng.2013.12.015.

URL http://linkinghub.elsevier.com/retrieve/pii/S0143816613003692

[49] N. Cornille, Mesure précise de formes et de déformations 3d d'objets observés au Microscope Électronique à Balayage (MEB), Ph.D. thesis, INSA de Toulouse (Jun. 2005). URL http://tel .archives-ouvertes.fr/tel-00166423

[50] M. Bornert, F. Brémand, P. Doumalin, J.-C. Dupré, M. Fazzini, M. Grédiac, F. Hild, S. Mistou, J. Molimard, J.-J. Orteu, L. Robert, Y. Surrel, P. Vacher, B. Wattrisse, Assessment of Digital Image Correlation Measurement Errors: Methodology and Results, Experimental Mechanics 49 (3) (2008) 353-370. doi:10.1007/s11340-008-9204-7.

URL http://link.springer .com/article/10.1007/s11340-008-9204-7

[51] H. W. Schreier, M. A. Sutton, Systematic errors in digital image correlation due to undermatched subset shape functions, Experimental Mechanics 42 (3) (2002) 303-310. doi:10.1007/BF02410987. URL http://link.springer .com/article/10.1007/BF02410987

[52] C. Efstathiou, H. Sehitoglu, J. Lambros, Multiscale strain measurements of plastically deforming polycrystalline titanium: Role of deformation heterogeneities, International Journal of Plasticity 26 (1) (2010) 93-106. doi:10.1016/j.ijplas.2009.04.006.

URL http://www.sciencedirect.com/science/article/pii/S0749641909000771

[53] J. Réthoré, A fully integrated noise robust strategy for the identification of constitutive laws from digital images, International Journal for Numerical Methods in Engineering 84 (6) (2010) 631-660. doi:10.1002/nme.2908.

URL http://doi.wiley.com/10.1002/nme.2908 